\title{
Erratum to: Integrodifference equations, Allee effects, and invasions
}

\author{
Mei-Hui Wang • Mark Kot • Michael G. Neubert
}

Published online: 8 February 2013

(C) Springer-Verlag Berlin Heidelberg 2013

\section{Erratum to: J Math Biol (2002) 44:150-168 DOI 10.1007/s002850100116}

The bottom curve in Fig. 4 was calculated or plotted incorrectly in the original publication of the article. The corrected figure and its caption are given below. This correction, however, does not affect the other results and conclusions in the original publication.

The authors wish to thank Michael Turelli, who wrote to say that Fig. 4 looked peculiar; he was right.

The online version of the original article can be found under doi:10.1007/s002850100116.

\section{M.-H. Wang}

Board of Education, Anne Arundel County Public Schools, Annapolis, MD 21401, USA

e-mail: meihuiwang2001@yahoo.com

M. Kot $(\square)$

Department of Applied Mathematics, Box 352420, University of Washington,

Seattle, WA 98195-2420, USA

e-mail: mkot@uw.edu

M. G. Neubert

Biology Department, Woods Hole Oceanographic Institution, Woods Hole, MA 02543-1049, USA

e-mail:mneubert@whoi.edu 


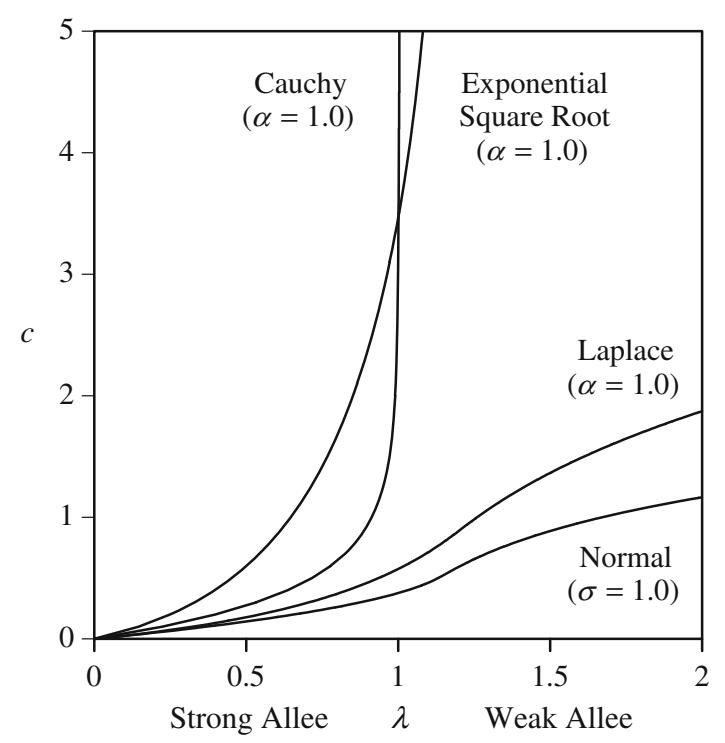

Fig. 4 The speed $c$ as a function of $\lambda$ for the Laplace $(\alpha=1.0)$, normal $(\sigma=1.0)$, exponential square root $(\alpha=1.0)$, and Cauchy $(\alpha=1.0)$ kernels and an Allee threshold of $a=0.5$. The $c$ urves were computed using iterative scheme (3.5) with $2^{14}$ mesh points, order $\lambda^{500}$, and upper limits of integration of $L=50, L=30, L=400$, and $L=6000$. The thin-tailed Laplace and normal distributions generate finite-speed traveling waves for all positive $\lambda$. The speed diverges to infinity for the fat-tailed exponential square root distribution for some $\lambda>1$ and for the fat-tailed Cauchy distributions at or near $\lambda=1$ 